\title{
Efectos económicos de la deserción en la gestión universitaria: el caso de una universidad pública chilena
}

\author{
LUIS AMÉSTICA-RIVAS* \\ ANDREA KING-DOMÍNGUEZ** \\ DANIELA SANHUEZA GUTIÉRREZ *** \\ VÍCTOR RAMÍREZ GONZÁLEZ****
}

Artículo de reflexión sobre los costes económicos de la deserción universitaria en la educación superior.

Recibido: 1 de mayo de 2020 - Evaluado: 27 de julio de 2020 - Aceptado: 8 de septiembre de 2020

Citar como: Améstica-Rivas, L., King-Domínguez, A. Sanhueza, D. y Ramírez, V. (2021). Efectos económicos de la deserción en la gestión universitaria: el caso de una universidad pública chilena. Hallazgos, 18(35), 209-231. https://doi.org/10.15332/2422409X.5772

\footnotetext{
* Doctor en Administración y Dirección de Empresas. Académico de la Universidad del Bío-Bío, Chile.

Correo electrónico: lamestica@ubiobio.cl ORCID: https://orcid.org/0000-0003-0482-0287

** Doctora en Administración y Dirección de Empresas. Académica de la Universidad del Bío-Bío, Chile.

Correo electrónico: aking@ubiobio.cl ORCID: https://orcid.org/0000-0002-1063-4336

*** Licenciada en Ciencias de Administración. Universidad del Bío-Bío, Chile. Correo electrónico: daniela.sanhueza1501@alumnos.ubiobio.cl ORCID: https://orcid.org/0000-0001-8200-0976

**** Licenciado en Ciencias de Administración. Universidad del Bío-Bío, Chile. Correo electrónico: victor.ramirez1501@alumnos.ubiobio.cl oRCID: https://orcid.org/0000-0002-1058-8878
} 


\section{Resumen}

La deserción es una realidad para las universidades en todo el mundo, especialmente en Latinoamérica. La deserción causa pérdidas de recursos difíciles de cuantificar, sobre todo para las universidades, cuya gestión financiera depende en gran parte de los ingresos provenientes de sus carreras de pregrado, en las que este fenómeno se da como un hecho cierto, sin existir una medida de valoración de los efectos económicos que crea para cada institución. En este contexto, se pretende cuantificar los costos de la deserción de una universidad estatal chilena, con base en sus datos académicos y financieros, entre 2016 y 2019. Se evidenció un abandono de 2900 estudiantes en el periodo, lo que representa un $7,67 \%$ promedio de deserción respecto a la matrícula total. El motivo principal de la deserción es la pérdida de carrera asociada a rendimiento académico y al hecho de que la mayoría de los estudiantes desertores tenía como fuente de financiamiento la beca de gratuidad, que representa un alto costo para el Estado. En términos monetarios, el costo de la deserción a 2019 alcanza los USD 23428 287,14.

Palabras clave: Chile; Deserción; Educación Superior; Economía de la Educación; Costes educativos. 


\title{
Economic effects of dropout in university management: the case of a Chilean public university
}

\begin{abstract}
Dropout is a reality for universities around the world, especially in Latin America. Dropout causes losses of resources that are difficult to quantify, especially for universities, whose financial management depend largely on income from their undergraduate careers, where this phenomenon is a fact, without there being a measure of assessment of the economic effects it creates for each institution. In this context, the aim is to quantify the dropout costs from a Chilean state university, based on its academic and financial data, between 2016 and 2019. A dropout of 2900 students was observed in the period, which represents a $7.67 \%$ average of dropout regarding the total enrollment. The main reason for dropping out is the loss of career associated with academic performance and the fact that most of the students who dropped out had free scholarship as source of funding, which represents a high cost for the State. In monetary terms, dropout cost by 2019 amounts to USD 23428 287.14.
\end{abstract}

Keywords: Chile; Dropout; Higher Education; Economics of Education; Educational Costs.

\section{Efeitos econômicos da desistência na gestão universitária: o caso de uma universidade pública chilena}

\section{Resumo}

A deserção é uma realidade para as universidades no mundo inteiro, especialmente na América Latina. Ela causa perdas de recursos difíceis de quantificar, sobre tudo para as universidades, cuja gestão financeira depende, em grande parte, dos ingressos provenientes de seus cursos de graduação, nos quais esse fenômeno ocorre como um fato certo, sem uma medida de avaliação dos efeitos econômicos criados para cada instituição. Nesse contexto, pretende-se quantificar os custos da desistência de uma universidade estadual chilena, com base em seus dados acadêmicos e financeiros, entre $2016 \mathrm{e}$ 2019. Foi evidenciado um abandono de 2900 estudantes no período, o que representa uma média de 7,67 \% de deserção com relação ao total de matriculados. O motivo principal é a reprovação associada ao desempenho acadêmico e ao fato de que a maioria dos estudantes desistentes tinha como fonte de financiamento bolsas de estudo, que representa um alto custo para o Estado. Em termos monetários, o custo da deserção em 2019 atingiu os USD\$ 23428 287,14.

Palavras-chave: Chile; Deserção; Ensino superior;Economia da educação; Custos educacionais. 


\section{Introducción}

La deserción educativa es un problema para las sociedades actuales, que genera efectos no solo en los estudiantes sino también en sus familias y en las instituciones que la experimentan. Los gobiernos, especialmente en América Latina, se han empeñado en buscar formas de disminuir y erradicar la deserción, especialmente en la educación superior. Sin embargo, aunque existen estadísticas, muchas instituciones de educación superior adolecen de una cuantificación de los recursos que dejan de percibir por efecto de la pérdida de estudiantes matriculados, a partir de una proyección de sus ingresos a futuro. Para la gestión de las universidades es de suma importancia conocer aquellos costos que afectan sus finanzas, para así tomar decisiones que fortalezcan su modelo educativo y adoptar políticas administrativas de forma eficiente y oportuna en un contexto de transparencia con su comunidad.

Bien se sabe que la deserción conlleva efectos financieros y organizativos en las instituciones educativas que van más allá del plano individual. En este sentido, la Organización para la Cooperación y el Desarrollo Económicos (OCDE) ha señalado que las mayores oportunidades de ingreso a la educación superior para los estudiantes no se acompañan de condiciones apropiadas que favorezcan el avance curricular, su retención, la calidad de su formación, graduación oportuna e inserción de los estudiantes más vulnerables, por lo que se siguen perpetuando las desigualdades e inequidades en la región (OCDE, 2009; Améstica, 2015). Más aún, disminuir la deserción y aumentar la graduación se ha convertido en un imperativo para los gobernantes en pos de mejoras respecto a la equidad y con el fin de enfrentar la exclusión social y económica de las poblaciones más vulnerables (King-Domínguez et ál., 2020).

Generalmente, los estudios sobre deserción se centran en los factores personales y externos que llevan al estudiante a desertar (Munizaga et ál., 2018). Sin embargo, el fenómeno es multicausal, por lo cual existe la posibilidad de poder abordarlo de distintas perspectivas. Ejemplo de ello, Titus (2006) estudia qué factores son determinantes de la deserción, y evidencia que el tipo de institución (privado versus público), ubicación (rural versus urbano) y tamaño (número de matrículas) de una universidad no influyen en la persistencia de los estudiantes. Es más, hay estudios que evidencian que las tasas de deserción en las universidades de carácter público son más altas que en las privadas (Gitto et ál., 2016). Este último factor invita a mirar con mayor detención las universidades estatales, en su papel de generadoras de bienes públicos, dado que lo que es una opción para la universidad privada es un 
imperativo para la institución estatal, que se debe a la sociedad como un todo (KingDomínguez et ál., 2020).

La realidad latinoamericana se caracteriza por una masificación creciente en el ingreso de estudiantes a la educación superior, por una alta heterogeneidad y por altas tasas de deserción. Esta última es una clara pérdida de eficiencia de la gestión de las instituciones de educación, con implicancias para las familias y el Estado, especialmente por la cantidad de recursos asignados como política del sector. Las altas tasas de deserción implican responsabilidades para la institución, no solo en lo que se refiere a investigar sus causas o diseñar estrategias para enfrentar el problema, sino también a cuantificar la magnitud del fenómeno y lo que esto significa en términos de costos para su gestión, pues algo no está funcionando como corresponde y los administradores universitarios deben tomar decisiones al respecto.

En este contexto, este trabajo pone de relieve y cuantifica monetariamente los efectos económicos que genera la deserción para las instituciones y el Estado, analizando una universidad estatal chilena como estudio de caso. Para ello, se realizó un estudio financiero, calculando los ingresos no percibidos por deserción en un periodo de cinco años de carreras de pregrado, como referencia de duración promedio de una carrera. El modelo supuso el uso de métodos de actualización de los montos a valor presente, considerando matrícula, deserción por carrera o programa, valores de arancel y costo de oportunidad en el periodo lectivo.

\section{Deserción y graduación en educación superior}

Améstica (2015), citando los trabajos seminales sobre deserción en la educación superior de Elías (2008), Georg (2009) y Tinto (1975), señala que la literatura ha establecido diferentes conceptos, causas y formas de entender el hecho de que un estudiante deje sus estudios o los abandone de manera temporal, parcial o total. En efecto, la deserción se entiende como un fenómeno complejo de estudiar, que no es fácil de precisar en términos conceptuales y operativos, y es investigado desde distintos enfoques y perspectivas (Castro-Montoya et ál., 2020), en el que se pueden distinguir dos focos: la decisión de abandonar los estudios (abandono o deserción) versus la decisión de permanecer en la institución (permanencia, persistencia o retención académica).

El estudio de la deserción encuentra su origen en los años setenta, y ejemplo de ello son los aportes de Spady, Tinto, Fishben y Ajzen (Améstica, 2015; Munizaga et ál., 
2018). Aunque las investigaciones iniciales de dichos autores abordan la deserción desde diversos enfoques, se esbozan cuatro factores que persisten hasta la actualidad: del propio individuo, académicos, socioeconómicos e institucionales. Asimismo, autores más contemporáneos han adaptado dichos modelos a la nueva realidad, especialmente realizando una combinación de los distintos factores y modelos. Tal como se señaló, la deserción es un fenómeno que no es posible explicar a partir de una sola o de múltiples causas, sino más bien de una interacción de factores. Para Elías (2008) y Stratton, et ál. (2008), el abandono puede ser de dos tipos: definitivo, adoptando el concepto de deserción, o por el contrario transitorio o de suspensión, en los casos en que los estudiantes llevan un tiempo sin matricularse y deciden retomar sus estudios, o también deciden cambiar sus estudios por redefinición de sus preferencias o vocación (Améstica, 2015).

Ahora bien, George (2009) establece que el abandono de una carrera o titulación por parte de un estudiante se diferencia de la ausencia de sus estudios, si bien lo considera un efecto posible que encuentra sus causas en factores académicos o extraacadémicos. Por lo tanto, no debe asimilarse a un problema solo de habilidades y de competencia del estudiante (Améstica, 2015). A nivel latinoamericano se destacan los trabajos de Himmel (2002), quien distingue la deserción voluntaria de la involuntaria, profundizando el análisis sobre el abandono de una carrera antes de alcanzar el título o grado y las posibilidades de retomar los estudios.

Por su parte, Blanco et ál. (2018) señalan que no se releva el tema del reingreso de los estudiantes al sistema de educación superior, lo cual afectaría el concepto clásico de lo que entiende por deserción. Es más, enfatizan que más de la mitad de los desertores reingresan, aunque sea cambiando de área de estudios, y alcanzan importantes tasas de titulación.

Un enfoque que ha prevalecido en el análisis son las condiciones socioculturales y económicas de los estudiantes como factor detonante de la deserción. La evidencia internacional muestra que se presentan menores tasas de finalización de estudios entre los estudiantes pertenecientes a minorías (Chen y Des Jardins, 2008; Chen, 2012), y a su vez "los estudiantes que abandonan los estudios superiores tienen una mayor probabilidad de pertenecer a familias más vulnerables y de menores niveles socioeconómicos" (Smith y Naylor, 2001; Vignoles y Powdthavee, 2009, citados en Améstica, 2015, p. 116), esto indica que existen mayores probabilidades de graduarse para estudiantes provenientes de familias más acomodadas. Donoso y Schiefelbein (2007) evidencian en sus estudios que las familias con bajos ingresos pueden ser el reflejo de un menor capital social y cultural, lo cual, por ende, repercute en que 
existan estudiantes menos preparados académicamente y con mayor riesgo de deserción. En ese sentido, la deserción resulta de la combinación de distintas variables y por su efecto, influyendo así en la integración social y académica del estudiante (Díaz, 2008).

En el nivel de la educación superior, algunos autores afirman que las medidas para enfrentar la deserción son de carácter aislado y de baja efectividad, ya que no se articulan en forma sistémica a una estrategia organizacional (Tinto, 2006). Kim y Kim (2018) señalan que, desde el punto de vista institucional, la deserción no solo representa un fracaso a la hora de adaptarse a la vida y al sistema universitario, sino que además prende una luz de alerta en lo que respecta a la prestación de servicios más apropiada para los estudiantes. Se evidencia que en los países pertenecientes a la OCDE, al año 2016, los estudiantes universitarios tenían un $49 \%$ de probabilidad de graduarse (Rodríguez et ál., 2018).

Al revisar la realidad latinoamericana, existe cierto consenso en reconocer que la deserción es una preocupación creciente entre los gobiernos, especialmente frente a lo que está sucediendo en la educación superior, debido a que constituye una fuente de ineficiencia, ya que limita las posibilidades de ampliación de cobertura, genera pérdidas económicas e impide la formación de recurso humano de avanzada (Castro-Montoya et ál., 2020). Ejemplo de ello es Colombia, donde se reconoce el impacto socioeconómico en las instituciones y en la población en general (Rueda et ál., 2020). Tanto es así que como programa ministerial se maneja el Sistema de Prevención y Análisis de la Deserción en las Instituciones de Educación Superior (sPADies). Por su parte, para el caso de México, Manríquez y Vásquez (2019) explican que, al igual que en otros países, la educación se ve trastocada por factores como la deserción y la reprobación.

En Argentina las tasas de matrícula universitaria son mayores que en Brasil, pero en este último la deserción en las universidades es menor, hecho que lo convierte en un sistema mucho más eficaz, con mayores tasas de graduación anual y mayor crecimiento en cantidad de graduados (Guadagni et ál., 2019). Sin duda la deserción tiene impactos en el bienestar y salud de los estudiantes, junto al costo económico que conlleva. Seminara y Aparicio (2019, citando a Lugo, 2013), establecen que, por año, América Latina y el Caribe pierden hasta 415 millones de dólares en algunos países, producto de la deserción universitaria. 


\section{Deserción y graduación en Chile}

Las políticas de ayuda financiera individual, en la forma de becas y créditos a los estudiantes, explica, en parte, el aumento creciente de la matrícula en la educación superior chilena (Blanco et ál., 2018), como también en Latinoamérica (Vargas y Heringer, 2017). Sin embargo, estos alentadores resultados también se han visto caracterizados por la existencia de altas tasas de abandono entre los estudiantes, que no les permite finalizar sus estudios y menos obtener su título universitario (Améstica, 2015). En Chile, al año 2009, el 58 \% de los estudiantes abandonaron la educación superior antes de completar un programa de estudios, porcentaje superior al promedio de 29,6 \% de la OCDE para el mismo periodo (OCDE, 2009; Améstica, 2015). La mejora en el acceso a la educación superior, en la última década, es coherente con la tendencia mundial. En términos de reducción de desigualdad, los resultados se muestran satisfactorios, dado que un mayor acceso a educación superior de estratos socioeconómicos más vulnerables ha permitido avanzar en la mejora de ingresos. Además, los estudiantes más vulnerables han aumentado cinco veces su ingreso en educación superior en los últimos veinte años. La evidencia local señala que el acceso a financiamiento estudiantil reduce las probabilidades de deserción, y que un buen diseño de instrumentos de apoyo y ayuda permite a los estudiantes mantenerse y finalizar sus estudios (Barrios, 2013).

En Chile se entiende que las condiciones actuales de la educación superior suponen desafíos crecientes para las instituciones, con un especial énfasis en la responsabilidad que significa atender al nuevo tipo de estudiante que se está incorporando, dado su perfil cultural y socioeconómico (Blanco et ál., 2018). Para Saldaña y Barriga (2010), dicho panorama se ha acrecentado en los últimos años, en la medida en que estudiantes provenientes de sectores más vulnerables y con menor capital social optan por universidades de baja selectividad y presentan, en general, mayor probabilidad de deserción. Asimismo, Améstica (2015, citando a Donoso et ál., 2010) reafirma las disparidades en términos de calidad, costos y duración de las carreras entre las instituciones, lo cual se convierte en un terreno fértil para la desigualdad, acentuando así algunos problemas transversales como la deserción y la eficiencia en la graduación; de ahí la necesidad imperiosa de incrementar la retención de estudiantes, fenómeno no visibilizado años atrás a pesar de las crecientes cifras.

Según los datos del Ministerio de Educación (Mineduc), al año 2017, en Chile persisten cifras llamativas, puesto que la tasa de retención promedio a primer año 
del sistema llega al $74 \%$, lo cual indica que de cada cuatro estudiantes que ingresan uno deserta del sistema. Análogo a lo señalado, mantenerse en el sistema representa para los estudiantes un desafío mayor que ingresar a este. Datos ministeriales (Mineduc, 2012) muestran tasas disímiles entre los tipos de instituciones de educación superior, manteniendo tasas de deserción sobre el $30 \%$ al tercer año (Améstica, 2015).

La deserción ha sido estudiada ampliamente en Chile en los últimos tiempos desde distintas ópticas, tanto cualitativas como cuantitativas: estudios enfocados a comparar con otras realidades de Latinoamérica (Vargas y Heringer, 2017); a identificar los factores causales de la deserción a nivel nacional y a hacer comparaciones territoriales a partir de las distintas corrientes y modelos predictivos (Donoso y Schiefelbein, 2007; Acuña et ál., 2010; Donoso et ál., 2010; Mineduc, 2012; Rau et ál., 2013; Barrios, 2013; Navarrete et ál.; 2013; Larracaou y Mizala, 2013; Horn et ál., 2014; Ramírez y Gradón, 2018; Blanco et ál., 2018); a identificar las variables explicativas en instituciones como estudio de caso (Canales y De los Ríos, 2009; Fernández et ál., 2009; Saldaña y Barriga, 2010); con aplicación a carreras o programas específicos (Reyes et ál., 2007; Gallegos et ál., 2018; Arancibia y Trigueros, 2018); e investigaciones que abordan ciertas experiencias locales a través de modelos conceptuales (Himmel, 2002; Díaz, 2008).

Una línea más innovadora en Chile son los estudios de eficiencia con modelo de Análisis Envolvente de Datos (DEA). Aunque las investigaciones de resultados de docencia son limitados, se observan trabajos especialmente orientados a la eficiencia de investigación, productividad cientifica o multiproducto (González-Araya y Vásquez, 2010; Ramírez y Alfaro, 2013; Muñoz, 2016). Un estudio reciente de DEA sobre la eficiencia en deserción y graduación aplicado a las universidades chilenas muestra la heterogeneidad del sistema, afirmando que el nivel de eficiencia nacional es de un 81,71\% (King-Domínguez et ál., 2020). A su vez, también se encuentran investigaciones a nivel de comparación de departamentos académicos como estudios de casos de deserción (Cáceres et ál., 2014). Sin embargo, no se tienen hasta el momento estudios de costeo y de efectos económicos de la deserción.

\section{Materiales y métodos}

Esta investigación se basa en el análisis de eventos dentro de un periodo de tiempo determinado para un grupo específico de población estudiantil que desertó del sistema. La población objetivo estuvo conformada por un universo total de 2900 estudiantes, 
desertores de una universidad chilena de carácter regional y de propiedad estatal, en el periodo 2016-2019. La oferta institucional es de 35 carreras de pregrado, con una matrícula anual total de 9500 estudiantes promedio. Para fines metodológicos, se entenderá la deserción como el abandono o interrupción de los estudiantes de su formación universitaria.

Los datos utilizados en la investigación fueron proporcionados por la institución, distinguiendo carrera, año de ingreso, año de la última matrícula, motivo de la deserción, valor de matrícula, valor del arancel de la carrera y forma de financiamiento o beneficio estudiantil asignado. En tanto, el número de matrícula de cada año de dicho periodo se obtuvo a partir de la información publicada por el Mineduc (2020).

En una primera aproximación se presentan algunos datos evolutivos de carácter descriptivo. El cálculo de la deserción se basó en la definición teórica de Himmel (2002), quien la define como el abandono o retiro del establecimiento. Según los datos institucionales, los motivos de deserción se pueden clasificar en: fallecimiento, cambio de carrera, renuncia definitiva y pérdida de carrera. Este último se entiende como el abandono obligatorio del estudiante por decisión de la institución, por diversos motivos de rendimiento académico. A su vez, aunque el cambio de carrera puede ser entendido como una alternativa que no supone una deserción definitiva del estudiante del sistema, se debe tener claridad de que esta opción implica recursos utilizados que no necesariamente retribuyen a la formación del estudiante, especialmente cuando no existe homologación de asignaturas aprobadas. Además de que, legalmente, la repitencia o el cambio de carrera conduce a la pérdida de becas.

En cuanto a la estimación de costos, respecto a la forma de financiamiento de la carrera de los estudiantes que desertaron, se establecieron tres tipologías: beca de gratuidad, beca de arancel y pago con recursos propios del estudiante, el cual aglutina el pago directo de las familias o la obtención de créditos estudiantiles que se contratan con el objetivo de devolverse una vez egresado. Para encontrar un valor aproximado, imputable a cada forma de financiamiento, se establecieron los siguientes supuestos:

a. Estudiantes que no poseen ningún beneficio estatal financian sus estudios con recursos propios.

b. El beneficio que se tenía al momento de desertar fue constante durante la trayectoria universitaria.

c. Los créditos estudiantiles (Crédito con Aval del Estado [CAE] o Fondo Solidario de Crédito Universitario [FSCU]) se consideraron como recursos propios, 
aunque el Ministerio de Educación de Chile los considera como parte de los beneficios estudiantiles. Ese enfoque se adoptó bajo el argumento de que, a largo plazo, es la persona que estudia quien se endeuda y debe pagar dicho crédito.

d. Estudiantes que ingresaron antes del año 2016, y contaban con beca de arancel total, se clasificaron como de gratuidad, dado el cambio de denominación.

Se consideró para el objeto del estudio el año de la última matrícula como base para el cálculo del año de la deserción. Teniendo en cuenta que este dato puede contar para el primer o el segundo semestre, se consideró que la deserción se produjo en ese mismo año $(t)$ para los matriculados en el primer semestre; en cambio, en el caso de los estudiantes cuya última matrícula fue en el segundo semestre, se considera que la deserción se produjo al año siguiente $(t+1)$.

Por consiguiente, para estimar la tasa de deserción anual $(t)$, se consideró el número de desertores $(t)$ con relación al número de matrícula del año anterior $(t-1)$, según ecuación (1).

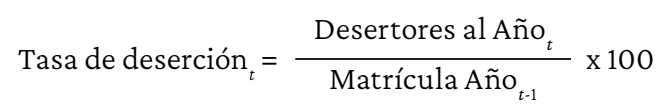

La estimación del costo monetario se basó en lo que desembolsaron los desertores en el periodo señalado, para lo cual se consideraron los valores históricos de arancel según el caso del estudiante, así como también el valor de la matrícula. El procedimiento consistió en determinar de cuánto fue la inversión monetaria que se costeó por cada año de estudio. Por lo tanto, el resultado esperado es la estimación del costo que generó un estudiante durante los años de estudio, que se asumen al año de deserción de cada estudiante (ecuación 2).

$$
\text { Costo deserción }_{i}=\sum \text { Arancel }_{t}+\sum \text { Matrícula }_{t}
$$

Donde $i$ : el desertor; $t$ : año de estudio.

Respecto a la forma de financiamiento de los estudios, se realizó la estimación de cómo y quién asumió dicho costo, para lo cual se consideró el beneficio que se registraba en la última matrícula. A partir de eso se trabajó bajo el supuesto que dicho beneficio fue otorgado desde el ingreso de la persona a la institución. 


$$
\text { Financiamiento de la deserción }_{i}=\Sigma\left(\text { Beneficios }_{t}\right) * n
$$

Donde $n$ : el número de años de estudios del desertor $i$.

En cuanto a los valores de los aranceles de las carreras, se consideró un valor promedio nominal de los distintos años (2016-2019), recogiendo el reajuste año a año aplicado por la institución, como un monto en pesos cada año. Asimismo, se realizó una conversión de pesos chilenos (CLP) a dólares estadounidenses (USD), al 30 de diciembre de cada año.

\section{Resultados}

La institución analizada presentó una matrícula promedio anual de pregrado de 9563 estudiantes desde el año 2015 al 2019. En la tabla 1 se muestra la evolución de la matrícula anual, además de que se explicita el número de deserciones por año (2016-2019), considerando el número de desertores de un periodo $(t)$ con relación al número de matrículas del periodo anterior $(t-1)$. Los resultados muestran que se alcanzaron los 725 estudiantes en promedio anual, lo cual representa un 7,67 \%, siendo el año 2017 el periodo que presentó una mayor tasa (10,02\%). En los dos últimos años se observa además una disminución en la tasa de deserción, lo cual tiene relación con el incremento en el número de matrículas, así como también con la reducción en los desertores. En lo que respecta al año 2019, la tasa de deserción estimada es del 6,18\%.

Tabla 1. Deserción de estudiantes de pregrado, 2016-2019.

\begin{tabular}{|l|c|c|c|c|c|c|}
\hline \multicolumn{1}{|c|}{ Año } & $\mathbf{2 0 1 5}$ & $\mathbf{2 0 1 6}$ & $\mathbf{2 0 1 7}$ & $\mathbf{2 0 1 8}$ & $\mathbf{2 0 1 9}$ & Promedio \\
\hline N. ${ }^{\circ}$ matrículas & 9439 & 9227 & 9575 & 9683 & 9890 & 9563 \\
\hline N. ${ }^{\circ}$ desertores & - & 706 & 925 & 671 & 598 & 725 \\
\hline$\%$ tasa deserción & - & $7,48 \%$ & $10,02 \%$ & $7,01 \%$ & $6,18 \%$ & $7,67 \%$ \\
\hline
\end{tabular}

Fuente: elaboración propia a partir del Informe Darca-UBв (2020).

El total de desertores en el periodo cubierto asciende a 2900 estudiantes de pregrado. Según se observa en la tabla, la variación entre los números de desertores es bastante volátil, pero decrece en los dos últimos años. 
Efectos económicos de la deserción en la gestión universitaria: el caso de...

Tabla 2. Evolución de estudiantes desertores, 2016-2019.

\begin{tabular}{|l|c|c|c|c|}
\hline \multicolumn{1}{|c|}{ Año } & $\mathbf{2 0 1 6}$ & $\mathbf{2 0 1 7}$ & $\mathbf{2 0 1 8}$ & $\mathbf{2 0 1 9}$ \\
\hline N. ${ }^{\circ}$ desertores & 706 & 925 & 671 & 598 \\
\hline Variación \% & - & $31,02 \%$ & $27,46 \%$ & $0,88 \%$ \\
\hline
\end{tabular}

Fuente: elaboración propia a partir del Informe Darca-UBв (2020).

Con relación a los motivos para desertar, como también para establecer las carreras más afectadas, se analizaron los 2900 desertores de acuerdo a los distintos motivos enunciados en la metodología (fallecimiento, cambio de carrera, renuncia definitiva y pérdida de carrera); en la tabla 3 se evidencia el porcentaje de cada uno de los motivos de abandono en el periodo de estudio. Se observa que los principales motivos de deserción estudiantil son la renuncia definitiva y la pérdida de carrera; en promedio, en el periodo señalado, la tasa deserción es de 34,82 \% y 52,44 \%, respectivamente. En menor proporción está el cambio de carrera, el cual presenta en promedio una tasa del 12,38 \%, siendo el año 2018 el periodo con un mayor nivel (17,44\%).

Tabla 3. Motivos de deserción 2016-2019.

\begin{tabular}{|l|c|c|c|c|c|}
\hline \multicolumn{1}{|c|}{ Año } & $\mathbf{2 0 1 6}$ & $\mathbf{2 0 1 7}$ & $\mathbf{2 0 1 8}$ & $\mathbf{2 0 1 9}$ & Promedio \\
\hline Fallecimiento & $0,42 \%$ & $0,22 \%$ & $0,45 \%$ & $0,33 \%$ & $0,36 \%$ \\
\hline Cambio de carrera & $14,59 \%$ & $12,32 \%$ & $17,44 \%$ & $5,18 \%$ & $12,38 \%$ \\
\hline Pérdida de carrera & $46,03 \%$ & $56,76 \%$ & $52,46 \%$ & $54,52 \%$ & $52,44 \%$ \\
\hline Renuncia definitiva & $38,95 \%$ & $30,70 \%$ & $29,66 \%$ & $39,97 \%$ & $34,82 \%$ \\
\hline
\end{tabular}

Fuente: elaboración propia a partir del Informe Darca-Uвв (2020).

En la tabla 4 se muestra la evolución de la deserción desde la perspectiva de las unidades académicas de la universidad, estructuradas administrativamente en facultades. En promedio, durante los últimos cuatro años, el 34,97\% de los desertores pertenecían a la Facultad de Ingeniería, siendo 2018 el año con la tasa de deserción más alta (38\%). Seguidamente está la Facultad de Ciencias Empresariales, cuyo promedio de desertores fue del $24,11 \%$ de los desertores. 
Tabla 4. Tasa de deserción por facultades, 2016-2019.

\begin{tabular}{|l|c|c|c|c|c|}
\hline \multicolumn{1}{|c|}{ Año } & $\mathbf{2 0 1 6}$ & $\mathbf{2 0 1 7}$ & $\mathbf{2 0 1 8}$ & $\mathbf{2 0 1 9}$ & Promedio \\
\hline $\begin{array}{l}\text { Arquitectura, Construcción } \\
\text { y Diseño }\end{array}$ & $11,90 \%$ & $8,54 \%$ & $11,03 \%$ & $11,87 \%$ & $\mathbf{1 0 , 8 3} \%$ \\
\hline Ciencias & $10,20 \%$ & $10,70 \%$ & $8,64 \%$ & $6,69 \%$ & $\mathbf{9 , 0 6} \%$ \\
\hline $\begin{array}{l}\text { Ciencias de la Salud } \\
\text { y de los Alimentos }\end{array}$ & $6,23 \%$ & $5,62 \%$ & $4,47 \%$ & $3,85 \%$ & $\mathbf{5 , 0 4} \%$ \\
\hline Ciencias Empresariales & $23,09 \%$ & $23,89 \%$ & $22,35 \%$ & $27,09 \%$ & $\mathbf{2 4 , 1 1 \%}$ \\
\hline Educación y Humanidades & $16,15 \%$ & $15,57 \%$ & $15,50 \%$ & $16,72 \%$ & $\mathbf{1 5 , 9 8} \%$ \\
\hline Ingeniería & $32,44 \%$ & $35,68 \%$ & $38,00 \%$ & $33,78 \%$ & $\mathbf{3 4 , 9 7 \%}$ \\
\hline
\end{tabular}

Fuente: elaboración propia a partir del Informe Darca-UBв (2020).

\section{Costo económico de la deserción}

El valor promedio de arancel en el periodo estudiado corresponde a USD 3323,2. La cuota básica por matrícula no presentó variación, por lo que su precio estuvo fijado en USD 123,6. Sin embargo, en el último año el valor del arancel tuvo un promedio de USD 3494,2. Dicho valor se encuentra sobre el promedio en el periodo de esta investigación, debido a que el valor de los aranceles fue reajustado año tras año.

La variación promedio del valor del arancel en pesos en el periodo de estudio es de 3,5 \%; la mayor tasa de variación de 4,2 \% ocurrió en el año 2019. Se destaca que dicha variación fue la misma para todos los aranceles de las carreras de pregrado. Además, las carreras con mayor valor arancelario al año 2019 fueron Arquitectura y Psicología, con un valor de USD 4274,7 y USD 4122,9, respectivamente. Sin embargo, el valor del arancel del resto de las carreras estuvo en el rango USD 2902,5-USD 3948,3.

En virtud del análisis se establece que el costo monetario acumulado en el periodo 2016-2019 (tabla 5), generado por la deserción estudiantil, asciende a USD 23428 275,2, siendo 2017 el año que registra un mayor costo de USD7 609 666,3, coincidente con las mayores tasas de deserción. Asimismo, 2017 tuvo la mayor variación en el periodo de estudio, con una fluctuación positiva del 64 \% en relación con el costo generado en 2016. Posteriormente, el costo tuvo una disminución del 24,8 \% 
al año siguiente, lo cual se explica por la variación negativa de la tasa de deserción, caso que también se observa en 2019, año en el que la tasa de deserción disminuyó y el costo bajó un $4,6 \%$.

Tabla 5. Evolución del costo monetario de la deserción, periodo 2016-2019.

\begin{tabular}{|l|c|c|c|c|}
\hline \multicolumn{1}{|c|}{ Año } & 2016 & 2017 & 2018 & 2019 \\
\hline Tasa de deserción \% & $7,48 \%$ & $10,02 \%$ & $7,01 \%$ & $6,18 \%$ \\
\hline Costo total USD & $\mathbf{4 6 4 0 1 4 5 , 3}$ & $\mathbf{7 6 0 9 6 6 6 , 3}$ & $\mathbf{5 7 2 2 ~ 0 1 7 , 2}$ & $\mathbf{5 4 5 6 4 4 6 , 5}$ \\
\hline
\end{tabular}

Fuente: elaboración propia a partir del Informe Darca-U Bв (2020).

Al distinguir el costo de deserción por motivo o causa (tabla 6), se puede evidenciar que el 52,4\% de los estudiantes que desertaron por motivo de pérdida de carrera alcanzaron un costo acumulado en los cuatro años de USD 13884 562,1. Lo anterior representa el 59,3\% del costo total por deserción. En tanto, el 34,8 \% de estudiantes que desertaron por renuncia (voluntad propia) tuvieron un costo de USD 6116 262,5, equivalente al $26,1 \%$ del costo total. El cambio de carrera representa un $14,2 \%$ del costo por deserción, con un monto de USD 3328 844,6. De igual forma, este último disminuyó un 70,6 \% en 2019 respecto al año anterior.

Tabla 6. Principales motivos de la deserción y su costo, periodo 2016-2019.

\begin{tabular}{|c|c|c|c|c|c|}
\hline \multirow[b]{2}{*}{ Año } & \multicolumn{5}{|c|}{ Año } \\
\hline & 2016 & 2017 & 2018 & 2019 & $\begin{array}{c}\text { Total } \\
\text { acumulado }\end{array}$ \\
\hline Fallecimiento & 24044,1 & 26259,2 & 23658,0 & 24644,8 & 98606,1 \\
\hline Cambio de carrera & 850454,3 & 1080262,6 & 1080101,8 & 318025,8 & 3328844,6 \\
\hline Pérdida de carrera & 2338146,8 & 4708975,8 & 3351752,1 & 3485687,3 & 13884562,1 \\
\hline Renuncia definitiva & 1427500,0 & 1794168,7 & 1266505,3 & 1628088,5 & 6116262,5 \\
\hline Total USD & 4640145,3 & 7609666,3 & 5722017,2 & 5456446,5 & 23428275,2 \\
\hline
\end{tabular}

Fuente: elaboración propia a partir del Informe Darca-U вв (2020). 


\section{Costo por tipo de financiamiento de la deserción}

Con respecto a la forma de financiamiento, existen diferentes recursos que inciden en la permanencia de los estudiantes universitarios en sus respectivas carreras. Bajo la premisa de que la deserción se financió por tres vías, tal como fue definido anteriormente, se constató que el 43,3\% fue financiado con beca de gratuidad, el 34,8 \% con beca de arancel y el 21,9 \% con recursos propios, los cuales incluyen pago directo y créditos estudiantiles (tabla 7).

Tabla 7. Financiamiento del costo de la deserción, periodo 2016-2019.

\begin{tabular}{|l|c|c|c|c|}
\hline \multicolumn{1}{|c|}{ Forma de financiamiento } & $\mathbf{2 0 1 6}$ & $\mathbf{2 0 1 7}$ & $\mathbf{2 0 1 8}$ & $\mathbf{2 0 1 9}$ \\
\hline Beca de gratuidad & 769113,28 & 3208376,52 & 3068129,20 & 3100777,04 \\
\hline Recursos propios & 2047543,59 & 2484536,74 & 1753589,38 & 1872461,28 \\
\hline Beca de arancel & 1823488,41 & 1916753,08 & 900298,59 & 483208,14 \\
\hline Total USD & $\mathbf{4 6 4 0 ~ 1 4 5 , 3}$ & $\mathbf{7 6 0 9 6 6 6 , 3}$ & $\mathbf{5 7 2 2 ~ 0 1 7 , 2}$ & $\mathbf{5 4 5 6 4 4 6 , 5}$ \\
\hline
\end{tabular}

Fuente: elaboración propia a partir del Informe Darca-UBв (2020).

Como se puede observar, la beca de gratuidad es la principal forma de financiamiento en el costo de la deserción, pues presentó un constante crecimiento durante el periodo de estudio. Se infiere que esto se debió a que una mayor cantidad de estudiantes pudo obtener este beneficio. Caso contrario sucede con las becas de arancel, ya que estas muestran un decrecimiento significativo en el periodo; en el año 2019 el costo asociado fue de USD 483208,14 . Finalmente, con respecto a los recursos propios, se puede analizar que esta forma de financiamiento no presentó mayor volatilidad en el periodo 2016-2019; incrementó porcentualmente en el último año en 6,8 \%, alcanzando un costo de USD 1872 461,28.

Con respecto a la beca de gratuidad, el $56 \%$ de los desertores tenían asignado este beneficio al momento de desertar, por lo que el 43,3\% del costo acumulado de la deserción se financió por esta política, lo cual equivale a USD 10146 396,03. En términos evolutivos, a partir de la instalación de esta forma de financiamiento en el sistema universitario aparece una variación significativa, particularmente en el año 2017 con relación al año anterior, con lo cual se infiere que resulta como producto del aumento de la cobertura y el reemplazo de otros mecanismos de financiamiento estatal. 
En cuanto a la beca de arancel, este beneficio financió el 21,9 \% del costo total acumulado de la deserción en el periodo estudiado, equivalente a USD 5123748,21 . Se puede observar un decrecimiento en los últimos años, siendo el año 2017 el periodo con mayor tasa de deserción.

Tal como se mencionó anteriormente, dentro de los recursos propios se consideran aquellos costos que asume el estudiante por no contar con algún beneficio estatal (gratuidad o beca de arancel), agrupando a quienes pagan directamente y que al momento de desertar estaban adscritos a un crédito estudiantil (CAE O FSCU). El costo total de la deserción por recursos propios asciende a USD 8158 130,98 acumulado al año 2019, lo cual equivale al 34,8 \% del costo total. Sin embargo, se observó que el costo no implica alta volatilidad en términos monetarios. Dentro de este costo, los créditos estudiantiles suman USD 948 134,62 en el periodo 2016-2019.

\section{Conclusiones}

La deserción en la educación superior ha sido estudiada desde distintos enfoques, especialmente en los últimos años, puesto que es un fenómeno de importancia para muchos gobiernos que tienen como objetivo central bajar dichas tasas, implementando políticas que ayuden a la permanencia y a una graduación más efectiva, disminuyendo la pérdida de estudiantes durante su periodo académico. En las mismas universidades, se han definido programas y planes que buscan generar las condiciones institucionales para una mayor retención de los estudiantes, y así disminuir la deserción, sobre todo en los primeros años.

Desde la perspectiva de la gestión universitaria, si bien existen múltiples estudios, no se cuenta con modelos o métodos para estimar económicamente los efectos de la deserción. Bien se sabe que existen costos para la sociedad en general, especialmente para quienes financian los estudios (familias, Estado y las propias instituciones). Sin embargo, el significado de la pérdida de estudiantes es una realidad que cada institución analiza en el seno de su propia gestión, generalmente asumiéndolo como un hecho cierto sin dimensionar la magnitud del problema en términos financieros, ni proyectar lo que significa tener menores ingresos futuros dentro de una estructura financiera con muchos costos fijos en su operación, en la que el gasto en recursos humanos es el más importante.

Se ha señalado en investigaciones anteriores que para una adecuada gestión económica de las universidades es necesario conocer su estructura de costos, por 
consiguiente, es indispensable generar información financiera que permita a los administradores universitarios la toma de decisiones racionales (Améstica-Rivas et ál. 2017). Aunque esta investigación es un estudio de caso, y sabiendo que es una realidad que no es posible extrapolar a todo el sistema, sí permite aproximarnos al significado que tiene, para distintos actores, la deserción en sí, en términos de relación costo-oportunidad desde una perspectiva del costeo.

Indistintamente de lo que se entiende por deserción en sus distintas aproximaciones, la tesis de Himmel (2002), definida en términos simples como de abandono involuntario, permite dar una base de análisis para estimar los costos de la deserción. Ejemplo de ello es que la pérdida de carrera representa más del 50 \% del fenómeno, siendo el motivo central de abandono de los estudios en la universidad analizada, lo que indica que la causa principal de deserción es el rendimiento académico del estudiante. Un segundo motivo es la renuncia definitiva, que es el abandono total de la institución por motivos personales.

Desde el punto de vista del costo de la deserción, se estima que el monto en cuatro años de operación alcanza los USD 23428 287,14. A su vez, considerando los aranceles que se cobran en las carreras y el número de 2900 desertores en el periodo, se puede señalar que el costo promedio por desertor se aproxima a USD 8078,7. Aunque en términos generales las universidades estatales poseen aranceles más bajos en comparación con las instituciones privadas, este valor de costo per cápita evidencia el alto costo de estudiar en Chile, país que posee uno de los aranceles más elevados del mundo desde hace muchos años (OCDE, 2009).

Desde la perspectiva del tipo de financiamiento de los estudios, la beca de gratuidad es el factor más importante del costo de deserción (43,3\%), pues exime al estudiante de todo tipo de pago, tanto matrícula como arancel, mientras que las llamadas "becas de arancel” (21,9 \%) cubren la totalidad o parte del arancel de las carreras. Ambas becas son otorgadas por el Estado, hecho que da cuenta del costo que implica para el presupuesto fiscal. Además, se analizan los desertores que financiaron sus carreras con recursos propios, incluidos los créditos estudiantiles, los cuales, inevitablemente, generan un endeudamiento a futuro de los estudiantes una vez egresados.

Aunque el estudio de la deserción se abocó específicamente al costo del arancel, no hay que olvidar que existen otros costos ocultos para que una persona pueda mantenerse en el sistema, especialmente los gastos que debe desembolsar una familia para financiar a sus hijos o las becas otorgadas por el Estado para aspectos de manutención, tales como: alojamiento, movilización, útiles y alimentación, entre otros. Independientemente de quién financie los estudios, es evidente que existe un 
costo de oportunidad para cualquiera de los actores cuando hay deserción. Y la responsabilidad es aún mayor para un Estado y especialmente para las instituciones que son financiadas con recursos estatales, ya que dicha financiación proviene de la recaudación de impuestos de los ciudadanos del país. Por lo tanto, el dimensionar el costo económico de un hecho que hoy afecta al sistema de educación superior es un deber para cualquier Gobierno, en la medida en que este debe rendir cuenta pública de lo que realiza y ser eficiente en el manejo de sus gastos, en pos de ayudar a mejorar los niveles de equidad y justicia de los sectores más vulnerables.

Es importante mencionar, por último, que esta perspectiva, aunque limitada a un aspecto económico-financiero, puede ser enriquecida con otras visiones que se encuentran en la literatura científica. Asimismo, se abren nuevas líneas interesantes de estudios para estimar el costo monetario que produce la deserción en la educación superior, desde una perspectiva nacional ligada a las tasas de graduación.

\section{Sobre los autores}

Luis Améstica-Rivas. Doctor en Administración y Dirección de Empresas por la Universidad Politécnica de Cataluña (UPC), España. Magíster en Administración, con mención en Finanzas, por la Universidad de Chile. Académico del Departamento de Gestión Empresarial, Facultad de Ciencias Empresariales, Universidad del Bío-Bío, Chile. Investigador del grupo de investigación en Dirección Universitaria de la UPC, España. Áreas de investigación: economía de la educación y finanzas de las empresas. Artículo recientemente publicado en coautoría: "Are local governments more efficient as the same political coalition governs? A study among Chilean municipalities” (2020).

Andrea King-Domínguez. Doctoranda en Administración y Dirección de Empresas por la Universidad Politécnica de Cataluña (UPC), España. Magíster en Administración, con mención en Gestión Financiera, por la Universidad de Chile. Académica del Departamento de Economía y Finanzas, Facultad de Ciencias Empresariales, Universidad del Bío-Bío, Chile. Investigadora asociada del grupo de investigación en Dirección Universitaria de la UPC, España. Área de investigación: finanzas corporativas y educación superior. Artículo recientemente publicado en coautoría: "Caracterización de la producción científica sobre clasificaciones de universidades. Un estudio bibliométrico desde 1988 a 2018” (2020).

Daniela Sanhueza Gutiérrez. Licenciada en Ciencias de Administración e Ingeniera Comercial, Programa Magíster en Gestión de Empresas (MGE), por la Universidad del Bío-Bío, Chile. Auxiliar de investigación en gestión universitaria, Uвв, Concepción, Chile. 
Víctor Ramírez González. Licenciado en Ciencias de Administración e ingeniero comercial, Programa Magíster en Gestión de Empresas (MGE), Universidad del Bío-Bío, Chile. Auxiliar de investigación en gestión universitaria, Uвв Concepción, Chile.

\section{Referencias}

Améstica Rivas, L. R. (2015). Retorno, financiamiento y deserción, factores clave de la educacción superior. Lecciones para la política públlica: el caso de Chile [Tesis doctoral, Universitat Politécnica de Catalunya (UPC), Departament d'Organització d'Empreses, España]. http:// hdl.handle.net/2117/95676

Améstica-Rivas, L., Llinas-Audet, X. y Escardíbul, J. O. (2017). Costos de la renovación curricular: una propuesta metodológica para la valorización económica de carreras universitarias. Formación universitaria, 10(1), 89-100. http://dx.doi.org/10.4067/ S0718-50062017000100010

Arancibia, R. y Trigueros, C. (2018). Aproximaciones a la deserción universitaria en Chile. Educação e Pesquisa, 44(e165743), 1-20. http://dx.doi.org/10.1590/s1678-4634201708165743

Barrios, A. (2013). Deserción universitaria en Chile: incidencia del financiamiento y otros factores asociados. Revista del Centro de Investigación Social de un Techo para Chile, (1), 59-72.

Blanco, C., Meneses, F. y Paredes, R. (2018). Más allá de la deserción: trayectorias académicas en la educación superior en Chile. Calidad en la Educación, (49), 137-187. http://dx.doi. org/10.31619/caledu.n49.579

Cáceres, H., Kristjanpoller, W. y Tabilo, J. (2014). Análisis de la eficiencia técnica y su relación con los resultados de la evaluación de desempeño en una universidad chilena. Innovar, 24(54), 199-217. https://doi.org/10.15446/innovar.v24n54.46720

Canales, A. y De los Ríos, D. (2009). Retención de estudiantes vulnerables en la educación universitaria. Revista Calidad en la Educación, 30(1), 50-83. doi: http://dx.doi.org/10.31619/ caledu.n30.173

Castro-Montoya, B. A., Manrique-Hernández, R. D., Gonzalez-Gómez, D. y Segura-Cardona, A. M. (2020). Trayectoria académica y factores asociados a graduación, deserción y rezago en estudiantes de programas de pregrado de una universidad privada de Medellín (Colombia). Formación universitaria, 13(1), 43-54. http://dx.doi.org/10.4067/ S0718-50062020000100043

Chen, R. (2012). Institutional characteristics and college student dropout risks: A multilevel event history analysis. Research in Higher Education, 53, 487-505. https://doi.org/10.1007/ s11162-011-9241-4

Chen, R. y Desjardins, S. (2008). Exploring the effects of financial aid on the gap in student dropout risks by income level. Research in Higher Education, 49(1), 1-18. https://doi. org/10.1007/s11162-007-9060-9 
Díaz, C. (2008). Modelo conceptual para la deserción estudiantil universitaria en chilena. Estudios Pedagógicos, 36(2), 65-86. https://scielo.conicyt.cl/scielo.php?script=sci_arttex t\&pid=S0718-07052008000200004

Donoso, S., Donoso, G. y Arias, O. (2010). Iniciativas de retención de estudiantes en Educación Superior. Revista Calidad en la Educación, (33), 15-61. http://dx.doi.org/10.31619/caledu. n33.138

Donoso, S. y Schiefelbein, E. (2007). Análisis de los modelos explicativos de retención de estudiantes en la universidad: una vision desde la desigualdad social. Estudios Pedagógicos, XXXIII(1), 7-27. http://dx.doi.org/10.4067/S0718-07052007000100001

Eckert, K. y Suénaga, R. (2015). Análisis de deserción-permanencia de estudiantes universitarios utilizando técnica de clasificación en minería de datos. Formación universitaria, 8(5), 03-12. http://dx.doi.org/10.4067/S0718-50062015000500002

Elias, M. (2008). Los abandonos universitarios: retos ante el Espacio Europeo de Educación Superior. Estudios sobre Educación, (15), 101-121.

Fernández, O., Martínez-Conde, M. y Melipillán, R. (2009). Estrategias de aprendizaje y autoestima. Su relacion con la permanencia y desercion universitaria. Estudios Pedagógicos, 35(1), 27-45. http://dx.doi.org/10.4067/S0718-07052009000100002

Ferrão, M. y Almeida, L. (2018). Multilevel modeling of persistence in higher education. Ensaio: Avaliação e Politicas Públicas em Educação, 26(100), 664-683. http://dx.doi.org/10.1590/ s0104-40362018002601610

Gallegos, J., Campos, N., Canales, K. y González, E. (2018). Factores determinantes en la deserción universitaria. Caso Facultad de Ciencias Económicas y Administrativas de la Universidad Católica de la Santísima Concepción (Chile). Formación Universitaria, 11(3), 11-18. http://dx.doi.org/10.4067/S0718-50062018000300011

Georg, W. (2009). Individual and institutional factors in the tendency to drop out of higher education: a multilevel analysis using data from the Konstanz Student Survey. Studies in Higher Education, 34(6), 647-661. https://doi.org/10.1080/03075070802592730

Gitto, L., Minervini, L. F. y Monaco, L. (2016). University dropouts in Italy: Are supply side characteristics part of the problem? Economic Analysis and Policy, (49), 108-116. https:// doi.org/10.1016/j.eap.2015.12.004

González-Araya, M. y Vásquez, G. (2010). Análisis de eficiencia y productividad de las universidades chilenas mediante análisis y encapsulamiento de datos. Aporte santiaguino, 3(2), 245-256.

Guadagni, A., Lima, G. y Boero, F. (2019). ¿Por qué hay dos domingos tan diferentes en Brasily la Argentina? Universidad de Belgrano-Centro de Estudios de la Educación Argentina (CEA), $8(87), 1-8$.

Gury, N. (2011). Dropping out of higher education in France: A micro-economic approach using survival analysis. Education Economics, 19(1), 51-64. https://doi. org/10.1080/09645290902796357 
Himmel, E. (2002). Modelos de análisis de la deserción estudiantil en la educación superior. Revista Calidad en la Educación, (17), 91-108. http://dx.doi.org/10.31619/caledu.n17.409

Horn, C., Santelices, M. y Catalán, X. (2014). Modeling the impacts of national and institutional financial aid opportunities on persistence at an elite Chilean university. Higher Education, 68(3), 471-488. https://doi.org/10.1007/s10734-014-9723-3

Kim, D. y Kim, S. (2018). Sustainable education: Analyzing the determinants of university student dropout by nonlinear panel data models. Sustainability, 10(4), 954,1-18. https:// doi.org/10.3390/su10040954

King-Domínguez, A., Backhouse Erazo, P. y Améstica-Rivas, L. (2020). Deserción y graduación. Midiendo la eficiencia de las universidades estatales en Chile. Mendive. Revista de Educación, 18(2), 1-11.

Manríquez, A. y Vázquez, E. (2019). Estrategias planteadas en el acompañamiento tutorial para evitar el rezago y deserción. Congreso CLABES IX, Bogotá D.C. Colombia, 1184-1191.

Mineduc (2012). Deserción en la educación superior en Chile. Serie Evidencias, 1(9), 1-12. www. mineduc.cl

Mineduc (2019). Informe retención de 1.er año de pregrado. Cohortes 2013-2018, 1-9. www.mineduc.cl

Munizaga, F., Cifuentes, M. y Beltrán, A. (2018). Retención y abandono estudiantil en la educación superior universitaria en América Latina y el Caribe: una revisión sistemática. Education Policy Analysis Archives, 26(60/61), 1-32. https://doi.org/10.14507/epaa.26.3348

Muñoz, D. (2016). Assessing the research efficiency of higher education institutions in Chile a data envelopment analysis approach. International Journal of Educational Management, 30(6), 809-825. https://doi.org/10.1108/IJEM-03-2015-0022

Organización para la Cooperación y el Desarrollo Económicos [OCDE] (2009). Tertiary Education in Chile. Reviews of National Policies for Education.

Ramírez, P. y Alfaro, J. (2013). Evaluación de la eficiencia de las universidades pertenecientes al Consejo de Rectores de las Universidades Chilenas: resultados de un análisis envolvente de Datos. Formación universitaria, 6(3), 31-38. http://dx.doi.org/10.4067/ S0718-50062013000300005

Ramírez, P. y Gradón, E. (2018). Predicción de la Deserción Académica en una Universidad Pública Chilena a través de la clasificación basada en Árboles de Decisión con Parámetros Optimizados. Formación universitaria, 11(3), 3-10. http://dx.doi.org/10.4067/ S0718-50062018000300003

Rau, T., Rojas, E. y Urzúa, S. (2013). Loans for Higher Education: Does the Dream Come True? (Working paper N. ${ }^{\circ}$ 19138) National Bureau of Economic Research. https://doi.org/10.3386/ w19138

Reisel, L. y Brekke, I. (2010). Minority Dropout in Higher Education: A Comparison of the United States and Norway Using Competing Risk Event History Analysis. European Sociological Review, 26(6), 691-712. https://doi.org/10.1093/esr/jcp045 
Rodríguez, A. B., Espinoza, J., Ramírez, L.J. y Ganga, A. (2018). Deserción universitaria: nuevo análisis metodológico. Formación universitaria, 11(6), 107-118. http://dx.doi.org/10.4067/ S0718-50062018000600107

Rueda Ramírez, S. M., Urrego Velásquez, D., Páez Zapata, E., Velásquez, C. y Hernández Ramírez, E. M. (2020). Perfiles de riesgo de deserción en estudiantes de las sedes de una universidad colombiana. Revista de Psicología, 38(1), 275-297. https://doi.org/10.18800/ psico.202001.011

Saldaña, M. y Barriga, O. (2010). Adaptación del modelo de deserción universitaria de Tinto a la Universidad Católica de la Santísima Universidad Católica de la Santísima Concepción, Chile. Revista de Ciencias Sociales, XVI(4), 616-628.

Seminara, M. y Aparicio, M. (2019). La deserción universitaria ¿un concepto equívoco? Revisión de estudios latinoamericanos sobre conceptos alternativos. Revista de Orientación Educacional, 32(61), 44-72.

Stratton, L., O'Toole, D. y Wetzel, J. (2008). A multinomial logit model of college stopout and dropout behavior. Economics of Education Review, 27(3), 319-331. https://doi. org/10.1016/j.econedurev.2007.04.003

Suárez-Montes, N. y Díaz-Subieta, L. (2015). Estrés académico, deserción y estrategias de retención de estudiantes en la educación superior. Revista de Salud Pública, 17(2), 300-313. http://dx.doi.org/10.15446/rsap.v17n2.52891

Taylor, B. y Harris, G. (2004). Relative efficiency among South African universities: A data envelopment analysis. Higher Education, (47), 73-89. https://doi.org/10.1023/ B:HIGH.0000009805.98400.4d

Tinto, V. (1975). Dropout from Higher Education: A theoretical synthesis of recent research. Review of Educational Research, (45), 89-125. https://doi.org/10.3102/00346543045001089

Tinto, V. (2006). Research and practice of student retention: what next? Journal of College Student Retention: Research, Theory and Practice, 8(1), 1-19. https://doi. org/10.2190/4YNU-4TMB-22DJ-AN4W

Titus, M. A. (2006). Understanding the influence of the financial context of institutions on student persistence at four-year colleges and universities. The Journal of Higher Education, 77(2), 353-375. https://doi.org/10.1080/00221546.2006.11778929

Vargas, H. y Heringer, R. (2017). Políticas de permanência no ensino superior público em perspectiva comparada: Argentina, Brasil e Chile. Education Policy Analysis Archives, (25), 1-33. http://dx.doi.org/10.14507/epaa.25.2799 